\title{
Impactos de uma Intervenção com Pais: O Desempenho Acadêmico e Comportamento das Crianças na Escola
}

\author{
Impacts of a Parent Intervention Program: Their Children's Academic \\ Achievement and Classroom Behavior
}

\author{
Fabiana Cia ${ }^{*}, a$, Elizabeth Joan Barham $^{a}$, \& Anne Marie Germaine Victorine Fontaine ${ }^{b}$ \\ ${ }^{a}$ Universidade Federal de São Carlos, São Carlos, Brasil \& ${ }^{b}$ Universidade do Porto, Porto, Portugal
}

\begin{abstract}
Resumo
Neste estudo avaliou-se o impacto de um programa de intervenção com os pais, sobre o desempenho acadêmico e o comportamento de crianças da $1^{\mathrm{a}}$ e $2^{\mathrm{a}}$ séries do Ensino Fundamental. Os participantes foram divididos em três grupos: Grupo Experimental 1 (GE1 - professores e 29 crianças cujos pais participaram da intervenção), Grupo Experimental 2 (GE2 - professores e 36 crianças cujas mães participaram da intervenção) e Grupo Controle (GC - professores e 34 crianças cujos pais e mães não participaram da intervenção). Antes e após o programa de intervenção, as crianças foram avaliadas utilizando-se o Teste de Desempenho Escolar (TDE); os professores preencheram um questionário aberto e o Social Skills Rating Scale -Versão para Professores (SSRS-P). Em comparação com o pré-teste, no pós-teste, as crianças do GE1 e do GE2 (mas não as do GC) apresentaram: (a) melhor desempenho acadêmico em leitura e na pontuação total do TDE, (b) resultados mais positivos no SSRS-P e, (c) um maior número de atributos positivos e menor número de atributos negativos, segundo as professoras.

Palavras-chave: Programa de intervenção; Desempenho acadêmico; Problemas de comportamento; Fase de transição; Envolvimento paterno.

Abstract

This paper presents the impacts of a parent intervention program on the academic performance and classroom behavior of their children. The participants of the study were either first or second grade students. There were three groups of participants: Experimental Group 1 (EG1 - 29 children whose fathers participated in the intervention program along with the children's teachers); Experimental Group 2 (EG2 - 36 children whose mothers participated in the intervention program along with the children's teachers); and Control Experimental Group (CG - 34 children, whose parents did not participate in the intervention program, and the children's teachers). Before and after the intervention program, the children were evaluated using the Academic Achievement Test (AAT) and their teachers completed an openended questionnaire as well as the teacher's version of the Social Skills Rating Scale (SSRS-T). In comparison with the pre-test, in the post-test the children in the EG1 and EG2 (but not in the CG) obtained: (a) higher overall scores in the AAT and in the reading sub-test; (b) more positive results in the SSRS-T; and (c) were rated by their teachers as presenting a greater number of positive attributes and a smaller number of negative attributes.

Keywords: Intervention program; Academic achievement; Behavior problems; Transition phase; Parent involvement.
\end{abstract}

É crescente o número de queixas escolares sobre os problemas de comportamento dos alunos (incluindo a violência escolar), que muitas vezes, estão relacionados com o baixo desempenho escolar. Diante dessa realidade, os Psicólogos Escolares precisam atuar para diminuir o encaminhamento desses estudantes para escolas

\footnotetext{
* Endereço para correspondência: Centro de Educação e Ciências Humanas, Departamento de Psicologia, Labor, Rodovia Washington Luís, Km 235, caixa postal 676, São Carlos, SP, Brasil, CEP 13565-905. E-mail: fabianacia@hotmail.com

Apoio Financeiro: Fundação de Amparo à Pesquisa do Estado de São Paulo (FAPESP).

Este trabalho é derivado da Tese da primeira autora.
}

ou serviços especiais (López, 2004; Martínez, 2007). Uma das estratégias é trabalhar com os pais, uma vez que os problemas de comportamento entre as crianças estão associados, principalmente, às características das interações familiares, à medida que os membros da família treinam diretamente esse padrão comportamental na criança. Quando a criança apresenta problemas, em geral, ambos os pais não usam reforçadores positivos contingentes às iniciativas pró-sociais dos seus filhos e fracassam no uso de técnicas disciplinares adequadas, que pudessem reduzir os comportamentos desviantes. Estas famílias se caracterizam pelo uso de disciplina severa e inconsistente, com pouco envolvimento parental, pouco monito- 
ramento e supervisão dos comportamentos das crianças (Cia, Pereira, Del Prette, \& Del Prette, 2006; Gomide, 2004; Pacheco, Alvarenga, Reppold, Piccinini, \& Hutz, 2005; Parke, 2004; Rocha \& Brandão, 2001). De fato, déficits nas habilidades necessárias para a manutenção da disciplina, no acompanhamento dos filhos, no uso adequado do reforço positivo, no emprego eficaz das técnicas de solução de problemas e na comunicação aberta podem colocar ambos os pais dentro da zona de alto risco de fracasso, em relação ao seu desempenho na educação e nos cuidados dos filhos (Olivares, Méndez, \& Ros, 2005).

A partir da década de 1990, os programas de intervenção passaram a se direcionar, cada vez mais, para o aumento da qualidade do relacionamento entre ambos os pais e seus filhos, diminuindo os efeitos adversos dos estressores que podem acometer as famílias e aumentando as habilidades e a sensibilidade parental para com os filhos (Carpenter, 1997). A realização de programas de intervenção com pais e mães é importante por questões éticas e pragmáticas. Em relação à questão ética, encontra-se o direito e a obrigação que ambos os pais têm de educar seus filhos. Ou seja, os programas devem dotálos da maior competência possível para promover a saúde da criança, prevenir ou resolver possíveis intercorrências no desenvolvimento infantil e facilitar a adaptação do filho ao seu contexto social, como também devem empoderá-los para que consigam resolver os problemas e educar os filhos, nas diferentes fases do desenvolvimento (Olivares et al., 2005; Williams \& Aiello, 2004).

No que diz respeito à questão pragmática, intervenções com a família são apontadas na literatura como tendo melhores resultados do que intervenções exclusivamente focadas nas crianças. Vários estudos realizados no Brasil, que avaliaram programas de intervenção para pais, apontaram benefícios, tais como: ampliação do repertório de habilidades sociais educativas e maior uso de reforçamento positivo dos pais (Barros \& Del Prette, 2007; Bolsoni-Silva, Del Prette, \& Del Prette, 2000; Bolsoni-Silva, Salina-Brandão, Versutti-Stoque, \& Rosin-Pinola, 2008; Bolsoni-Silva, Silveira, \& Marturano, 2008), diminuição dos problemas de comportamento (Bolsoni-Silva, Silveira, et al., 2008; Pinheiro, Haase, Del Prette, Amarante, \& Del Prette, 2006) e melhora no desempenho acadêmico das crianças (Barros \& Del Prette, 2007).

Duch (2005) analisou os benefícios para ambos os pais e seus filhos, em diferentes programas de intervenção. Estes programas tinham por objetivo aumentar o envolvimento dos pais e mães no processo educacional e no desenvolvimento dos filhos e oferecer oportunidades para ambos os pais darem seguimento aos estudos e conquistarem um emprego, visando o empoderamento da família. De modo geral, a participação no programa veio a contribuir com: uma diminuição no estresse parental, uma melhoria no bem-estar emocional da criança e de seus familiares, maior estabilidade socioeconômica da família, uma melhoria nas atitudes parentais e um aumento na conscientização de ambos os pais quanto aos seus direitos. No entanto, o autor aponta que a redução efetiva nos riscos para o desenvolvimento infantil e para o fracasso escolar depende do engajamento dos pais e das mães no programa, bem como, de características familiares.

Apesar da importância dos programas de intervenção para a família, nota-se que a maioria deles é direcionada apenas para a mãe, sendo escassas as intervenções em que os pais participam, ou mesmo aquelas direcionadas apenas para a figura paterna (Coley, 2001; Fabiano, 2007; Lamb, 1997; Taylor \& Daniel, 2000). Com base nos poucos trabalhos sobre programas de intervenção que envolveram o pai em idade adulta, verifica-se que os pais (homens) que participaram destes programas apresentaram ganhos diversos, com impactos positivos para toda a família.

Por exemplo, Fagan e Iglesias (1999) avaliaram um dos programas do Head Start, que envolvia atividades para pais afro-americanos e latino-americanos de poder aquisitivo baixo, cujos filhos estavam iniciando as atividades escolares (idade média de 4,5 anos), objetivando melhorar o envolvimento do pai nas atividades acadêmicas do filho e, consequentemente, melhorar a adaptação da criança à escola. Para analisar a eficácia do programa, os autores compararam 55 homens que participaram da intervenção com 41 homens que não participaram. Todos foram avaliados em dois momentos (préteste e pós-teste), levantando dados sobre: o envolvimento do pai em casa, o envolvimento do pai em leitura para os filhos, o desempenho da criança na escola e as habilidades sociais da criança. Comparando os homens que participaram por mais tempo da intervenção, com os que não participaram ou que participaram com menor intensidade, os principais resultados mostraram que: os filhos apresentaram maior repertório de habilidades sociais e maior motivação nos estudos, os homens apresentaram maior interação com os filhos, leram mais para os filhos, apresentaram menos problemas conjugais, se mostraram mais envolvidos nas interações em sala de aula com os filhos e se tornaram mais motivados em auxiliar seus filhos nas atividades acadêmicas.

A participação dos homens em um programa de intervenção torna-se ainda mais importante considerando que várias pesquisas apontam uma relação entre a qualidade do relacionamento paterno, o desempenho acadêmico e os problemas de comportamento dos filhos. Mais especificamente, o suporte emocional e estímulo para o desenvolvimento que os pais oferecem aos filhos, as habilidades sociais educativas dos pais, a qualidade da supervisão parental e o envolvimento do pai nas atividades escolares e diárias do filho, contribuem para que o filho estude com maior freqüência fora do contexto escolar, tenha maior motivação nos estudos e freqüente mais as aulas, o que resulta em melhor desempenho acadêmico 
(Cia, D’Affonseca, \& Barham, 2004; Flouri \& Buchanan, 2003; Hong \& Ho, 2005). Por outro lado, quando os pais têm baixo repertório de habilidades sociais educativas com o filho (Flouri, 2005; Hill et al., 2004), utilizam práticas educativas punitivas e autoritárias (Atzaba-Poria, Pike, \& Deater-Deckard, 2004; Cia, Pamplin, \& Del Prette, 2006; Davidov \& Grusec, 2006), não oferecem suporte emocional e afeto (Crean, 2008; Eisenberg et al., 2005; Fletcher, Steinberg, \& Williams-Wheeler, 2006) e não são calorosos com o filho (Davidov \& Grusec, 2006; Fletcher et al., 2006), os filhos têm maior probabilidade de apresentar problemas de comportamento externalizantes e internalizantes.

Devido às mudanças recentes nos papéis masculinos e femininos, o trabalho de intervenção com o pai deve ser enfatizado, pois, apesar dos homens, nos tempos atuais, estarem mais envolvidos na educação e nos cuidados diários com os seus filhos do que nas gerações anteriores, algumas das diferenças entre os gêneros persistem, pois as mulheres ainda estão sendo as principais responsáveis por estas tarefas (dedicando duas vezes mais tempo do que os homens, em média), embora exista pouca diferença no tempo dedicado ao trabalho remunerado por pais e mães que trabalham fora (Ahmed \& Bould, 2004; Gravena, 2006; Nascimento, 2007; Raley, Mattlingly, \& Bianchei, 2006). Ao mesmo tempo, sabe-se claramente que o envolvimento paterno é extremamente importante, graças aos estudos recentes que enfocaram a díade pai e filho e mostraram sua influência no desenvolvimento infantil. Assim, existe uma oportunidade importante para intervir com os pais, para maximizar a qualidade do relacionamento entre estes e seus filhos e, conseqüentemente, propiciar melhores condições para o desenvolvimento infantil.

Considerando a importância das práticas parentais para o desempenho acadêmico e para os comportamentos dos filhos e a escassez de intervenções realizadas com pais, no contexto brasileiro, este estudo teve por objetivo avaliar a eficácia de um programa de intervenção para pais, por meio de melhorias nos comportamentos e no desempenho acadêmico de escolares, no contexto de sala de aula. A eficácia de duas estratégias de intervenção foi comparada. Ambas pretendiam aumentar o investimento paternal na educação dos filhos, mas a primeira envolveu diretamente os pais (homens), exigindo a sua presença nas diversas sessões de intervenção, enquanto a segunda pretendia influenciar indiretamente o comportamento paterno, pelo intermédio das mães, graças à presença destas nas sessões de intervenção.

\section{Método}

\section{Participantes}

Para medir os impactos do programa de intervenção para pais sobre o desenvolvimento da criança, foram contatadas duas escolas municipais (que foram sele- cionadas via indicação da Secretaria Municipal) e uma escola estadual (que foi selecionada pelo interesse da diretora na realização da pesquisa), localizadas em um município no interior do estado de São Paulo.

Foram utilizados como critérios para ser participante: a criança viver com ambos os pais (biológicos ou não), a criança estar alfabetizada (considerou-se alfabetizada a criança que obteve um mínimo de cinco pontos em escrita e aritmética e 14 pontos em leitura num teste de desempenho acadêmico, descrito abaixo), o pai estar empregado e se interessar em participar da intervenção. Os pais que frequentaram as sessões da intervenção, junto com seus filhos, formaram o Grupo Experimental. Quando os pais quiseram, mas não puderam participar da intervenção, em função de incompatibilidade de horários, mas as suas esposas tinham interesse e horários disponíveis para participar da intervenção, estes pais e seus filhos formaram o Grupo Experimental 2. O Grupo Controle foi formado pelos pais e esposas que quiseram participar da intervenção, mas que não puderam. Com estes três grupos, foi possível separar os efeitos das duas estratégias de intervenção usadas das mudanças produzidas por demais variáveis (história, maturação da criança e mudanças de atitudes de ambos os pais de acordo com a idade da criança).

Essa pesquisa contou com a participação de 99 crianças: 29 no Grupo Experimental 1, 36 no Grupo Experimental 2 e 34 no Grupo Controle. As crianças apresentaram média de idade de oito anos, sendo que 78,8\% estavam na $2^{a}$ série e $21,2 \%$ na $1^{a}$ série do Ensino Fundamental. A maioria das crianças era de classe socioeconômica C e B2 (segundo o Critério Brasil, 2006). Nas sessões de intervenção, oferecidas em vários dias e horários diferentes, participaram 29 pais do Grupo Experimental 1, e 34 mães do Grupo Experimental 2 (duas mães tinham filhos gêmeos). Além disso, havia 34 pais no Grupo Controle. A média de idade dos pais era de 35 anos e a das mães de 33 anos. Ressalta-se que para as três escolas foram enviados convites para todos os pais/ mães de crianças da $1^{\mathrm{a}}$ e $2^{\mathrm{a}}$ séries do Ensino Fundamental, para participarem da intervenção.

Além disso, participaram deste estudo 20 professoras, com média de idade de 40 anos, que davam aula para as crianças incluídas no estudo. Destas, seis professoras lecionavam para a $1^{\mathrm{a}}$ série e 14 para a $2^{\mathrm{a}}$ série do Ensino Fundamental. Em média, estas professoras lecionavam há 14 anos; apenas duas delas estavam cursando o $3^{\circ}$ grau (para completar sua formação em pedagogia) e o restante já tinha o $3^{\circ}$ grau completo (15 professoras formadas em pedagogia, uma em letras, uma em história e outra em pedagogia e letras).

Para medir os impactos do programa de intervenção para pais sobre o desenvolvimento da criança, utilizouse um delineamento pré/pós-teste com grupo controle não equivalente. Segundo Cozby (2002), nesse delineamento existe um grupo experimental e um grupo controle, 
embora os participantes não fossem aleatoriamente selecionados, nem distribuídos aleatoriamente pelas condições, pois foram formados espontaneamente, de acordo com a disponibilidade do pai ou da mãe participar da intervenção, em horários previamente estipulados. Mesmo no caso dos grupos não serem equivalentes, é possível avaliar o impacto da intervenção, comparando os escores do pré-teste e do pós-teste.

\section{Instrumentos}

Crianças. Teste de Desempenho Escolar - TDE $(\alpha=$ 0,79). Para obter o índice do desempenho escolar das crianças, foi utilizado o Teste de Desempenho Escolar ([TDE], Stein, 1994), instrumento com propriedades psicométricas adequadas (alta confiabilidade interna) que avalia as capacidades fundamentais para o desempenho escolar. Este teste foi concebido para a avaliação de escolares da $1^{\underline{a}}$ a $6^{\underline{a}}$ séries do Ensino Fundamental e é composto por três subtestes: (a) escrita, que envolve a escrita do nome próprio e de 34 palavras isoladas, apresentadas sob a forma de ditado; (b) aritmética, que requer a solução oral de três problemas e a solução, por escrito de mais 35 operações aritméticas e, (c) leitura, que requer o conhecimento de 70 palavras, isoladas do contexto.

Professores. Social Skills Rating System - SSRS - Versão para Professores $(\alpha=0,81)$. Esta versão (elaborada por Gresham \& Elliott, 1990, validado para o nosso contexto por Bandeira, Del Prette, Del Prette, \& Magalhães, 2009) foi aplicada para avaliar a percepção das professoras quanto à existência e intensidade de problemas de comportamento internalizantes e externalizantes e o desempenho acadêmico das crianças, sendo composta por duas escalas tipo Likert: (a) 18 itens em que a professora assinala qual a freqüência (nunca, algumas vezes e muito freqüentemente) que a criança emite cada um dos comportamentos problema (sendo que esses itens se dividem em dois fatores: comportamentos problemáticos externalizantes e comportamentos problemáticos internalizantes) e, (b) nove itens perguntando sobre o desempenho do aluno em leitura e matemática, sua motivação geral, a participação dos seus pais, seu funcionamento intelectual e comportamento geral em classe (permitindo classificar o aluno em uma das seguintes categorias: entre os $10 \%$ piores, $20 \%$ piores, $40 \%$ médios, $20 \%$ bons ou entre os $10 \%$ ótimos).

Avaliação do Desempenho Acadêmico e dos Comportamentos dos Alunos. Elaborado com base em instrumentos já existentes (Omeño, 2004), este questionário é composto por duas questões abertas, em que a professora aponta quais são os comportamentos negativos e positivos do aluno, assim como avalia o desempenho acadêmico dos mesmos.

\section{Procedimentos de Coleta de Dados}

Para que os pais dessem seu consentimento para sua própria participação e a dos seus filhos na pesquisa e para que os professores dessem seu consentimento para a sua participação, foram entregues os Termos de Consentimento Livre e Esclarecido (TCLE). A coleta de dados seguiu o mesmo procedimento no pré-teste e no pósteste (antes e após a intervenção com os pais). Após o consentimento dos pais, um contato com as crianças foi estabelecido, para explicar as atividades que desenvolveriam e visando o estabelecimento do rapport. Em outro momento, foi aplicado nas crianças o Teste de Desempenho Escolar - TDE (que requer uma aplicação individual, com tempo médio para a aplicação de 45 minutos). As professoras da $1^{\underline{a}}$ e $2^{\underline{a}}$ séries do Ensino Fundamental foram contatadas, a fim de preencherem o questionário "Avaliação do desempenho acadêmico e dos comportamentos dos alunos" e o instrumento SSRSVersão para Professores. Para esclarecer as dúvidas das mesmas, as informações foram fornecidas individualmente, em sala de aula. A pesquisadora se atentou para que as professoras não soubessem em qual dos três grupos cada criança estava inserida (GE1, GE2 ou GC) para diminuir a probabilidade das expectativas das professoras interferirem na avaliação dos seus alunos.

\section{Intervenção}

A intervenção focou o aprimoramento das habilidades sociais educativas dos pais para com seus filhos e o ensino de práticas parentais favorecedoras de comportamentos pró-acadêmicos, por parte dos filhos. Esta intervenção teve por objetivo orientar os participantes, de forma didática, sobre: (a) os fundamentos da análise do comportamento; (b) a necessidade de motivar seus filhos a se comportarem bem e a terem comportamentos adequados aos estudos; (c) a identificação dos determinantes de comportamentos desadaptativos dos filhos; (d) a aplicação, no dia a dia, dos procedimentos básicos de modificação do comportamento; e (e) as crenças e os valores dos pais. A intervenção se baseou no pressuposto de que as crianças precisam ser reforçadas de modo frequente, contingente, intenso, diferenciado e sistemático e que os pais precisam agir como modelos adequados para seus filhos, se apoiando em intervenções realizadas por Caballo e Simón (2005), Cooper e Cooper (2005), Del Prette e Del Prette (2005), Fagan e Iglesias (1999) e Pinheiro et al. (2006), dentre outros teóricos, que seguem uma abordagem cognitivo-comportamental.

A intervenção com os pais ocorreu em um período de três meses, num total de 12 sessões: Programa de intervenção para pais, O papel paterno, Como manter e instalar comportamentos adequados no seu filho? Reconhecendo e extinguindo comportamentos inadequados do seu filho, Treinando comportamentos paternos empáticos, Leitura do ambiente e análise funcional do comportamento infantil, O elogio e o feedback positivo dos pais para os filhos, Auxiliando no desempenho acadêmico do filho - Parte 1 e 2, Desenvolvendo a capacidade de se expressar, Impondo limites e solicitando mudanças de comportamento do filho e Encerramento do grupo. Realizaram-se encontros semanais de 90 a 120 minutos de 
duração. Em todas as sessões, foi solicitada tarefa de casa ao final do encontro (exceto na sessão 12) e essas tarefas foram sempre retomadas e discutidas na próxima sessão. Durante as sessões, anotavam-se os relatos de tarefa de casa dos pais, junto com outros exemplos e comentários que surgissem. Além disso, eram entregues materiais explicativos impressos (folders, folhetos, exemplos ilustrativos, entre outros) para os participantes levarem para casa e consultarem, quando necessitavam. A participação dos pais nas sessões foi em média de 94,6\% (variando entre $100 \%$ a $90,5 \%$ de presença). Durante o programa de intervenção, os pais poderiam solicitar atendimentos individuais com a pesquisadora, em caso de dificuldades que não pudessem solucionadas durante os encontros. Além disso, foi estabelecido com os pais que haveria reposição das sessões para aqueles que precisavam faltar, antes de ocorrer a próxima sessão (os pais poderiam repor a falta participando num outro horário, porque havia vários grupos paralelos na mesma escola). Ao final de cada sessão, dois pais, em média, recebiam atendimento individual. No total, foram oferecidos oito grupos de pais: (a) Escola 1: Grupo 1, realizado à tarde, composto por dois pais e duas mães; Grupo 2, realizado à noite, composto por dois pais e quatro mães; (b) Escola 2: Grupo 1, realizado de manhã, composto por dois pais e três mães; Grupo 2, realizado à tarde, composto por dois pais e quatro mães; Grupo 3, realizado à noite, composto por sete pais e seis mães; (c) Escola 3: Grupo 1, realizado à noite, composto por nove pais e seis mães; Grupo 2, realizado à tarde, composto por quatro mães; Grupo 3, realizado à noite, composto por cinco pais e cinco mães.

\section{Procedimentos de Análise de Dados}

A pontuação dos dados obtidos no TDE foi realizada com base nos procedimentos apresentados no manual. Nos subtestes de escrita, leitura e aritmética foram dados um ponto para cada resposta correta. O escore bruto de cada subteste e o escore bruto total de todo o TDE foram convertidos por meio de uma tabela, com base na idade dos alunos, para se chegar à classificação do desempenho: superior, médio e inferior, para cada série escolar. Para comparar o desempenho acadêmico das crianças, considerando apenas um grupo, foi necessário combinar os escores das crianças da $1^{\underline{a}}$ e $2^{\underline{a}}$ séries do Ensino Fundamental no TDE. Sempre que é preciso combinar dados de dois grupos independentes, com distribuições normais, mas médias diferentes, podese subtrair ou somar um valor fixo a todos os escores de um dos grupos, para transpor a média para o mesmo valor do segundo grupo, sem afetar a forma da distribuição dos escores do primeiro grupo (Hays, 1981). Sendo assim, optou-se por transformar os escores das crianças da $1^{\text {a }}$ série para se ter uma distribuição equivalente aos das crianças da $2^{\underline{a}}$ série. Para tanto, foram acrescentados cinco pontos nos escores de cada criança da $1^{\text {a }}$ série em aritmética, oito pontos em escrita, quatro pontos em leitura e 17 pontos no escore total. Os dados do SSRS-Versão para Professores foram analisados com base no manual.

Por meio do questionário Avaliação do desempenho acadêmico e dos comportamentos dos alunos, foram obtidos dados qualitativos. Os dados qualitativos foram submetidos a uma análise de conteúdo e foram computadas a freqüência de respostas em cada uma das categorias. Para comparar os dados obtidos entre os três grupos (GE1, GE2 e GC), no pré-teste e no pós-teste, utilizou-se o ANOVA, MANOVA e o Qui-quadrado (usando o software SPSS- for Windows).

\section{Resultados}

Por meio de testes estatísticos (ANOVAe Qui-quadra$d o$ ), verificou-se que os dados sociodemográficos dos participantes não diferiram entre os três grupos e entre as três instituições de ensino. Na Tabela 1, compara-se os resultados obtidos no pré-teste e no pós-teste, entre as crianças do GE1, GE2 e GC, em relação ao seu desempenho acadêmico. Tanto na avaliação pré-teste, quanto pós-teste, o desempenho acadêmico de cada grupo de crianças (avaliado usando o Teste de Desempenho Escolar e o SSRS-Versão para Professores) estava na faixa média, de acordo com as normas estabelecidas para cada instrumento. Para verificar se existiam diferenças estatisticamente significativas no desempenho acadêmico das crianças, entre os dois grupos experimentais, no início da intervenção, ou seja, no pré-teste, realizou-se uma análise estatística (ANOVA), em que se comparou o valor das diferenças entre o pré-teste e o pós-teste nos GE1 e GE2 (subtrai o valor do pré-teste sob o valor do pósteste). A análise indicou que não houve diferenças estatisticamente significativas, em relação ao padrão de desempenho acadêmico destes dois grupos.

No intervalo entre o pré-teste e o pós-teste, observaram-se alterações favoráveis e significativas na pontuação no subteste de leitura e escore total do TDE, entre as crianças do GE1 $(F(1,28)=11,7, p<0,01$ - leitura; $F(1,28)=12,9, p<0,01-$ total do TDE) e do GE2 $(F(1,35)$ $=9,94, p<0,01-$ leitura; $F(35)=6,40, p<0,05-$ total do TDE). Observou-se o mesmo padrão, comparando o desempenho acadêmico dos alunos segundo as avaliações realizadas pelas professoras; entre o pré-teste e o pósteste, em que se verificou uma mudança positiva e significativa na pontuação obtida pelas crianças do GE1 $(F(1,28)=37,5, p<0,001)$ e do GE2 $(F(1,35)=17,6$, $p<0,001)$, como mostram os dados da Tabela 1 . Nenhuma mudança significativa foi observada no GC. Na Tabela 2 os problemas de comportamento internalizantes e externalizantes foram comparados, segundo a avaliação das professoras (com base no SSRS - Versão para Professores), no pré-teste e no pós-teste, entre as crianças do GC, GE1 e GE2. 
Tabela 1

Desempenho Acadêmico das Crianças no Pré-Teste e no Pós-Teste: Comparação do GE1, GE2 e GC Subtestes-TDE*

\begin{tabular}{|c|c|c|c|c|c|c|c|c|c|c|c|c|}
\hline & \multicolumn{12}{|c|}{ Grupo } \\
\hline & \multirow{3}{*}{$\begin{array}{r}\begin{array}{r}\text { Pré- } \\
\text { teste }\end{array} \\
\text { Média/ } \\
D P\end{array}$} & \multicolumn{3}{|c|}{ Experimental $1 \quad(N=29)$} & \multicolumn{4}{|c|}{ Experimental $1(N=34)$} & \multicolumn{4}{|c|}{ Controle $(N=36)$} \\
\hline & & $\begin{array}{l}\text { Pós- } \\
\text { teste }\end{array}$ & & anova & $\begin{array}{l}\text { Pré- } \\
\text { teste }\end{array}$ & $\begin{array}{l}\text { Pós- } \\
\text { teste }\end{array}$ & Mar & lova & $\begin{array}{l}\text { Pré- } \\
\text { teste }\end{array}$ & $\begin{array}{l}\text { Pós- } \\
\text { teste }\end{array}$ & Manc & \\
\hline & & $\begin{array}{r}\text { Média/ } \\
D P\end{array}$ & $F$ & $\mathrm{gl}$ & $\begin{array}{r}\text { Média/ } \\
D P\end{array}$ & $\begin{array}{r}\text { Média/ } \\
D P\end{array}$ & $F$ & $\mathrm{gl}$ & $\begin{array}{r}\text { Média/ } \\
D P\end{array}$ & $\begin{array}{r}\text { Média/ } \\
D P\end{array}$ & $F$ & gl \\
\hline Aritmética & $\begin{array}{r}11,7 \\
(03,7)\end{array}$ & $\begin{array}{r}12,9 \\
(04,5)\end{array}$ & $\mathrm{ns}$ & ns & $\begin{array}{r}12,4 \\
(03,1)\end{array}$ & $\begin{array}{r}12,7 \\
(02,6)\end{array}$ & ns & ns & $\begin{array}{r}12,1 \\
(03,5)\end{array}$ & $\begin{array}{r}11,3 \\
(04,2)\end{array}$ & ns & ns \\
\hline Escrita & $\begin{array}{r}23,7 \\
(08,2)\end{array}$ & $\begin{array}{r}24,9 \\
(08,6)\end{array}$ & ns & ns & $\begin{array}{r}28,7 \\
(05,0)\end{array}$ & $\begin{array}{r}29,3 \\
(05,6)\end{array}$ & ns & ns & $\begin{array}{c}24,92 \\
(13,4)\end{array}$ & $\begin{array}{r}6,6 \\
(07,8)\end{array}$ & ns & ns \\
\hline Leitura & $\begin{array}{r}59,1 \\
(16,8)\end{array}$ & $\begin{array}{r}67,1 \\
(06,3)\end{array}$ & $11,7 * *$ & 1,28 & $\begin{array}{r}65,6 \\
(05,9)\end{array}$ & $\begin{array}{r}67,9 \\
(03,2)\end{array}$ & $9,94 * *$ & 1,35 & $\begin{array}{r}56,5 \\
(16,8)\end{array}$ & $\begin{array}{r}61,1 \\
(11,6)\end{array}$ & ns & ns \\
\hline Escore total & $\begin{array}{r}94,9 \\
(24,8)\end{array}$ & $\begin{array}{l}104,9 \\
(16,3)\end{array}$ & $12,9 * *$ & 1,28 & $\begin{array}{l}106,6 \\
(11,5)\end{array}$ & $\begin{array}{r}109,9 \\
(08,5)\end{array}$ & $6,40 *$ & 1,35 & $\begin{array}{r}93,5 \\
(25,2)\end{array}$ & $\begin{array}{r}99,0 \\
(18,4)\end{array}$ & ns & ns \\
\hline $\begin{array}{l}\text { SSRS-Versão } \\
\text { para professores** }\end{array}$ & $\begin{array}{r}33,3 \\
\quad \quad(09,5)\end{array}$ & $\begin{array}{r}37,8 \\
(07,6)\end{array}$ & $37,5^{* *}$ & 1,28 & $\begin{array}{r}36,8 \\
(07,5)\end{array}$ & $\begin{array}{r}40,1 \\
(05,7)\end{array}$ & $17,6^{* *}$ & 1,35 & $\begin{array}{r}34,4 \\
(09,4)\end{array}$ & $\begin{array}{r}36,9 \\
(08,0)\end{array}$ & ns & ns \\
\hline
\end{tabular}

Nota: *Pontuação máxima em aritmética = 38 (Escore médio $=10-13)$; pontuação máxima em escrita = $35($ Escore médio = 20-26); pontuação máxima em leitura $=70$ (Escore médio $=58-66)$ e pontuação máxima total $=143$ (Escore médio = 87-105). ** Pontuação máxima $=45$ (Escore médio $=27,72-42,42)$. ${ }^{*} p<0,05 ; * *<<0,01 ; * * *<<0,001$; ns = não apresenta diferenças estatisticamente significativas.

Tabela 2

Pontuação Média nas Medidas de Problemas de Comportamento das Crianças, segundo as Professoras, no Pré-Teste e no Pós-Teste: Comparação do GE1, GE2 e GC

\begin{tabular}{|c|c|c|c|c|c|c|}
\hline & \multicolumn{6}{|c|}{ Grupo } \\
\hline & \multicolumn{2}{|c|}{ Experimental $1 \quad(N=29)$} & \multicolumn{2}{|c|}{ Experimental $1(N=34)$} & \multicolumn{2}{|c|}{ Controle $(N=36)$} \\
\hline & $\begin{array}{r}\text { Pré-teste } \\
\text { Média/DP }\end{array}$ & $\begin{array}{r}\text { Pós-teste } \\
\text { Média/DP }\end{array}$ & $\begin{array}{r}\text { Pré-teste } \\
\text { Média/DP }\end{array}$ & $\begin{array}{r}\text { Pós-teste } \\
\text { Média/DP }\end{array}$ & $\begin{array}{r}\text { Pré-teste } \\
\text { Média/DP }\end{array}$ & $\begin{array}{r}\text { Pós-teste } \\
\text { Média/DP }\end{array}$ \\
\hline $\begin{array}{l}\text { CPI-Comportamentos } \\
\text { problemáticos internalizantes }\end{array}$ & $\begin{array}{r}4,24 \\
(3,44)\end{array}$ & $\begin{array}{r}3,97 \\
(3,27)\end{array}$ & $\begin{array}{r}3,92 \\
(2,82)\end{array}$ & $\begin{array}{r}3,53 \\
(2,51)\end{array}$ & $\begin{array}{r}3,79 \\
(3,57)\end{array}$ & $\begin{array}{r}3,56 \\
(3,83)\end{array}$ \\
\hline CPE - Comportamentos & 11,5 & 9,89 & 9,69 & 8,61 & 9,47 & 9,03 \\
\hline problemáticos externalizantes & $(7,35)$ & $(7,18)$ & $(6,14)$ & $(5,96)$ & $(6,94)$ & $(6,95)$ \\
\hline CPT - Comportamentos & 15,7 & 13,9 & 13,6 & 12,1 & 13,3 & 12,6 \\
\hline problemáticos total & $(9,60)$ & $(9,29)$ & $(7,56)$ & $(6,85)$ & $(9,47)$ & $(9,43)$ \\
\hline
\end{tabular}

Nota. CPI (média professores = 0,00-3,24); CPE: (média professores =0,25-7,85); CPT: (média professores =0,87-8,97).

De acordo com a avaliação das professoras, as crianças apresentaram problemas de comportamento acima da média, considerando as normas estabelecidas com a amostra de referência para este instrumento (Bandeira et al., 2009), não havendo alterações significativas entre o pré-teste e o pós-teste. Na Tabela 3, o número de atributos positivos e negativos atribuídos aos alunos pelas professoras foi comparado no pré-teste e no pós-teste, para o GE1, GE2 e GC.

Nota-se um aumento significativo no número de atributos positivos mencionados em relação às crianças do $\operatorname{GE} 1(F(1,28)=40,8, p<0,001)$ e do $\operatorname{GE} 2(F(2,35)=10,0$, $p<0,01)$. Ao comparar os atributos negativos mencionados pelas professoras em relação a cada criança, antes e depois da intervenção, nota-se que houve uma diminuição significativa no número de atributos negativos citados para as crianças do GE1 $(F(1,56)=8,91, p<0,01)$ e do GE2 $(F(1,70)=13,4, p<0,01)$. Ao comparar as diferenças entre o pré-teste e pós-teste, nos três grupos, verificou-se, usando a análise ANOVA, que o GE1 apresentou diferença significativa no número de atributos positivos que as professoras atribuíram aos alunos, entre as fases de pré-teste e pós-teste, quando comparado com o GE2 e GC $(F(2,96)=23,1, p<0,001)$. Na 
Cia, E., Barham, E. J. \& Fontaine, A. M. G. V. (2010). Impactos de uma Intervenção com Pais: O Desempenho Acadêmico e Comportamento das Crianças na Escola.

Tabela 3

Número de Atributos Positivos e Negativos atribuídos os Alunos pelas Professoras, no Pré-Teste e no Pós-Teste: Comparação do GE1, GE2 e GC

\begin{tabular}{|c|c|c|c|c|c|c|c|c|c|c|c|c|}
\hline & \multicolumn{12}{|c|}{ Grupo } \\
\hline & \multicolumn{4}{|c|}{ Experimental $1(N=29)$} & \multicolumn{3}{|c|}{ Experimental 1} & & \multicolumn{4}{|c|}{ Controle $(N=34)$} \\
\hline & \multirow{2}{*}{$\begin{array}{l}\text { Pré- } \\
\text { teste }\end{array}$} & \multirow{2}{*}{$\begin{array}{l}\text { Pós- } \\
\text { teste }\end{array}$} & \multicolumn{2}{|c|}{ Manova } & \multirow{2}{*}{$\begin{array}{l}\text { Pré- } \\
\text { teste }\end{array}$} & \multirow{2}{*}{$\begin{array}{l}\text { Pós- } \\
\text { teste }\end{array}$} & & & \multirow{2}{*}{$\begin{array}{l}\text { Pré } \\
\text { teste }\end{array}$} & \multirow{2}{*}{$\begin{array}{l}\text { Pós } \\
\text { teste }\end{array}$} & \multicolumn{2}{|c|}{ Manova } \\
\hline & & & $F$ & $\mathrm{gl}$ & & & $F$ & $\mathrm{gl}$ & & & $F$ & $\mathrm{gl}$ \\
\hline $\begin{array}{l}\text { Média do N. de atributos } \\
\text { positivos do aluno. } \\
\text { Média do N. de atributos }\end{array}$ & 2,10 & 2,76 & $40,8^{* * *}$ & 1,28 & 2,00 & 2,22 & $10,0^{* *}$ & 2,35 & 2,00 & 1,94 & ns & ns \\
\hline negativos do aluno. & 1,24 & 1,00 & $8,91 * *$ & 1,56 & 1,39 & 1,11 & $13,4 * *$ & 1,70 & 1,00 & 0,94 & ns & ns \\
\hline
\end{tabular}

Nota: $* * p<0,01 ; * * * p<0,001 ; \mathrm{ns}=$ não apresenta diferenças estatisticamente significativas.

Tabela 4

Porcentagem de Alunos que Melhoraram o Desempenho Acadêmico, do Pré-Teste para o Pós-Teste, segundo as Professoras: Comparação do GE1, GE2 e GC

\begin{tabular}{|c|c|c|c|c|c|c|c|c|c|}
\hline & \multicolumn{9}{|c|}{ Grupo } \\
\hline & \multicolumn{3}{|c|}{ Experimental $1(N=29)$} & \multicolumn{3}{|c|}{ Experimental $2(N=36)$} & \multicolumn{3}{|c|}{ Controle $(N=34)$} \\
\hline & \multirow{2}{*}{$\begin{array}{l}\text { Pré- } \\
\text { teste }\end{array}$} & \multirow{2}{*}{$\begin{array}{l}\text { Pós- } \\
\text { teste }\end{array}$} & \multirow{2}{*}{$\frac{\text { Teste de } \chi^{2}}{\chi^{2} \mathrm{gl}}$} & \multirow{2}{*}{$\begin{array}{l}\text { Pré- } \\
\text { teste }\end{array}$} & \multirow{2}{*}{$\begin{array}{l}\text { Pós- } \\
\text { teste }\end{array}$} & Teste de $\chi^{2}$ & \multirow{2}{*}{$\begin{array}{l}\text { Pré- } \\
\text { teste }\end{array}$} & \multirow{2}{*}{$\begin{array}{l}\text { Pós- } \\
\text { teste }\end{array}$} & Teste de $\chi^{2}$ \\
\hline & & & & & & $\begin{array}{ll}\chi^{2} & \mathrm{gl}\end{array}$ & & & $\overline{\chi^{2}} \mathrm{gl}$ \\
\hline $\begin{array}{l}\text { Desempenho de } \\
\text { médio à muito } \\
\text { bom/excelente }\end{array}$ & $55,2 \%$ & $82,8 \%$ & $5,16^{*} 1$ & $61,1 \%$ & $86,1 \%$ & $5,79 *$ & $55,9 \%$ & $73,5 \%$ & ns \\
\hline
\end{tabular}

Nota: ${ }^{*} p<0,05 ; \mathrm{ns}=$ não apresenta diferenças estatisticamente significativas.

Tabela 4, compara-se a porcentagem de alunos que aumentou seu rendimento acadêmico, segundo as professoras, no GE1, GE2 e GC.

Comparando o desempenho acadêmico das crianças, no pré-teste e no pós-teste, segundo as professoras, a porcentagem de crianças que tinha um desempenho de médio a muito bom/excelente, aumentou significativamente no GE1 $\chi^{2}(1)=5,16, p<0,05$ e no GE2 $\chi^{2}(1)=5,79$, $p<0,05$. As professoras não observaram uma melhoria no desempenho acadêmico das crianças do GC.

\section{Discussão}

Inicialmente é interessante ressaltar que os dados sociodemográficos verificados para as crianças, seus pais e seus professores, que captam algumas das características pessoais e condições de vida que podem influenciar no desenvolvimento das crianças, não diferiram entre os três grupos e entre as três instituições de ensino, de forma que pode-se ter mais confiança que as mudanças no desempenho acadêmico e nos comportamentos das crianças sejam decorrentes da participação dos pais e das mães no programa de intervenção e não pela influência de diferenças entre os grupos ligadas às variáveis intervenientes.
Em relação à interpretação dos resultados que avaliaram diferentes aspectos do desenvolvimento das crianças, é importante lembrar que estes alunos encontravamse numa fase de transição, precisando se acostumar com diferentes demandas da vida escolar. Desta forma, o bom desempenho acadêmico e a boa adaptação social ao ambiente escolar representam duas demandas centrais enfrentadas por crianças nesta faixa etária e, quando seu desempenho nestas duas áreas é bem sucedido, os ganhos que obtenham no ambiente escolar passam a agir como fatores de proteção para seu desenvolvimento global (Aspesi, Dessen, \& Chagas, 2005; Marturano, 2004). Em relação às crianças que participaram deste estudo, do lado negativo, os índices de problemas de comportamento internalizantes e externalizantes estavam acima da média, nas fases de pré-teste e continuaram acima da média no pós-teste, segundo os resultados obtidos com o SSRS-Versão para Professores. Esses dados são preocupantes, pois a literatura aponta uma relação entre problemas de comportamento, com baixo rendimento acadêmico, pouco relacionamento com os pares e problemas emocionais (D'Avila-Bacarji, Marturano, \& Elias, 2005; Del Prette \& Del Prette, 2005; Dessen \& Szelbracikowski, 2004; Dunn, Cheng, O’Connor, \& Bridges, 2004; López, 2004). Fica evidente que elevados 
problemas de comportamento podem repercutir desfavoravelmente no desenvolvimento futuro (Bongers, Koot, Ende, \& Verhulst, 2004; Coley, Morris, \& Hernandez, 2004) e que o programa de intervenção não alterou significativamente este fator de risco. Contudo, o desempenho acadêmico das crianças (avaliados pelo TDE e pelo SSRS-Versão para Professores) estava na média, segundo as normas estabelecidas com a amostra de referência de cada instrumento e as crianças nos dois grupos cujos pais receberam a intervenção demonstraram ganhos significativos no seu desempenho acadêmico, podendo contribuir para a promoção de seu desenvolvimento.

Mais especificamente, ao comparar os dados das crianças, entre o pré e pós-teste, quanto ao desempenho acadêmico, pôde-se verificar que as crianças do GE1 e GE2 apresentaram um aumento significativo, no que diz respeito à pontuação em leitura, no escore total do TDE, na avaliação do desempenho acadêmico do SSRSVersão para Professoras e na porcentagem delas avaliadas pelas professoras como apresentando um desempenho acadêmico médio a excelente. A intervenção abordou temáticas específicas ao desempenho acadêmico dos filhos (Sessões 8 e 9), mas além disso incluiu alguns temas que também podem ter favorecido esses resultados, como, por exemplo: (a) os comportamentos parentais diante dos bons e maus comportamentos dos filhos, incluindo estabelecimento de regras, já que o estudar também é um comportamento; (b) o treino da empatia, assertividade e da habilidade de oferecer feedback aos filhos, os quais auxiliam os pais a modelarem os comportamentos das crianças, além de facilitar o estabelecimento de um relacionamento de maior confiança e respeito entre pais e filhos e, (c) a importância de estabelecer uma interação freqüente e de qualidade com os filhos, incluindo o brincar, já que o mesmo auxilia no desenvolvimento cognitivo das crianças, uma vez que o sucesso acadêmico não depende apenas da participação dos pais no ambiente escolar dos filhos, mas também das interações que estabelecem com os filhos (Machado, 2007).

Outro fator que pode ter contribuído para este impacto foi o fato dos pais, em várias sessões, solicitarem informações sobre a metodologia de ensino das professoras e de como resolver problemas dos filhos em sala de aula. Isso demonstra a carência de informações que existe entre os pais e ajuda a compreender como medidas simples de orientação, como ter um espaço na escola para que pais, professores e gestores possam trocar experiências e informações acerca do processo de ensino dos escolares, poderiam ajudar a reduzir problemas de aprendizagem de escolares.

É importante, também, refletir sobre os motivos pelos quais esta intervenção não resultou em melhorias comportamentais por parte das crianças, em sala de aula, segundo a avaliação das professoras. As contingências comportamentais no ambiente familiar são diferentes do ambiente escolar, sendo que a intervenção trabalhou apenas com a modificação do primeiro ambiente e não do segundo. Assim, ressalta-se a necessidade de se avaliar intervenções específicas para as demandas sociais escolares, ou mesmo um trabalho de sensibilização com as professoras, em paralelo com o programa realizado com os pais. No entanto, os impactos da intervenção nesta dimensão de adaptação social não são totalmente ausentes, uma vez que o número de atributos positivos atribuídos aos alunos dos dois grupos experimentais aumentou, segundo as professoras, e o número de atributos negativos diminuiu.

A similaridade dos resultados obtidos nos dois grupos experimentais também foi um achado importante. Isso parece apontar que a realização de treinamentos com um dos pais traz impactos positivos no desenvolvimento dos filhos e que tanto os pais quanto as mães são importantes figuras para a maximização do desenvolvimento infantil.

Acredita-se que um fator que contribuiu muito para o impacto significativo deste programa de intervenção foi a alta freqüência dos pais nas sessões. Alguns fatores podem ter contribuído para isso: (a) os participantes tinham a oportunidade de repor faltas (havia pelo menos duas ofertas de cada sessão por semana, em horários diferentes, por instituição de ensino) e, em casos de não poder ir nesses horários, poderiam marcar reposição; (b) houve uma preocupação de atender as necessidades dos pais, incorporando outras temáticas nas sessões; (c) a oferta de atendimentos individuais após cada sessão; (d) contato telefônico para lembrar os participantes do próximo encontro e, (e) sorteio de brindes, ao término do programa (usando uma estratégia motivacional sugerido por Caballo \& Simón, 2005; Conte, 2001; Pinheiro et al., 2006). Os participantes também avaliaram todas as sessões, o que possibilitou a realização de alterações nos procedimentos adotados nas mesmas, para atender às necessidades de todos. Ressalta-se que se tratava de uma população com poder aquisitivo médio e médio-baixo, o que favoreceu a adesão dos pais em um programa de intervenção.

Dentro da questão de adesão, é importante destacar a alta participação dos homens neste programa de intervenção. Primeiramente, tratou-se de uma intervenção direcionada para os mesmos, pois a maioria dos programas é direcionado apenas para as mães, mesmo aqueles que permitem a participação dos homens (Coley, 2001; Lamb, 1997; Taylor \& Daniel, 2000). Isso vem a demonstrar que os pais estão se comportando como esperado pela nova paternidade, ou seja, não estão tendo apenas o papel de provedor financeiro, mas estão colaborando, junto com a esposa, nos cuidados e na educação dos filhos (Brandth \& Kvande, 2002; Cia \& Barham, 2008; Matta $\&$ Knudson-Martin, 2006; Tiedje, 2004). 


\section{Considerações Finais}

A curto prazo, o programa de intervenção para pais mostrou-se eficaz, melhorando o desempenho acadêmico e alguns comportamentos (atributos) das crianças, no contexto escolar. Neste sentido, um programa de intervenção com esta estrutura deve nortear o trabalho de outros pesquisadores e especialistas na área, intervindo com diferentes amostras populacionais.

Quanto aos procedimentos e técnicas adotados no programa de intervenção, ainda serão necessárias análises mais detalhadas para identificar quais seriam os mais promissores, para garantia da sua eficácia. Apesar de algumas técnicas terem sido empregadas, no presente estudo, para garantir a permanência dos pais no programa de intervenção, ressalta-se a importância de aprimorar estas técnicas e de investigar outras opções, para determinar quais são os métodos mais eficazes para conseguir altas taxas de adesão. Sendo assim, são indispensáveis replicações futuras com a mesma clientela e com crianças de diferentes faixas etárias e necessidades, para que a proposta de intervenção seja refinada.

O impacto do programa de intervenção deve ser analisado com cautela. Apesar de este estudo ter sido conduzido em três instituições de ensino, com populações diferenciadas, contribuindo para a generalização dos resultados, esse mesmo fator pode contribuir para o menor controle das variáveis, uma vez que se tratam de populações e escolas diferenciadas. Também foi o caso que a seleção dos participantes e a sua alocação nos grupos não foram aleatórias. Além disso, os oito sub-grupos de participantes não eram homogêneos: eles não tinham um número igual de participantes (variando de quatro a 15), assim como, nem todos os grupos tinham números iguais de homens e mulheres. Também é importante lembrar que os participantes eram voluntários, porque nem sempre as pessoas com maiores dificuldades são aquelas com condições para participar de intervenções desta natureza. Assim, para garantia da validade interna do estudo, o mesmo deveria ser replicado, utilizando um delineamento experimental.

\section{Referências}

Ahmed, S. S., \& Bould, S. (2004). "One able daughter is worth 10 illiterate sons": Reframing the patriarchal family. Journal of Marriage and Family, 66, 1332-1341.

Aspesi, C. C., Dessen, M. A., \& Chagas, J. F. (2005). A ciência do desenvolvimento humano: Uma perspectiva interdisciplinar. In M. A. Dessen \& A. L. C. Costa (Eds.), A ciência do desenvolvimento humano: Tendências atuais $e$ perspectivas futuras (pp. 19-36). Porto Alegre, RS: Artmed.

Atzaba-Poria, N., Pike, A., \& Deater-Deckard, K. D. (2004). Do risk factors for problem behavior act in a cumulative manner? An examination of ethnic minority and majority children through an ecological perspective. Journal of Child Psychology and Psychiatry, 45(4), 707-718.
Bandeira, M., Del Prette, Z. A. P., Del Prette, A., \& Magalhães, T. (2009). Validação das escalas de habilidades sociais, comportamentos problemáticos e competência acadêmica (SSRS-BR) para o Ensino Fundamental. Psicologia: teoria e prática, 25(2), 271-282.

Barros, S. K. S. N., \& Del Prette, A. (2007). Um treinamento de habilidades sociais para pais pode beneficiar os filhos na escola? Revista da Sociedade de Psicologia do Triângulo Mineiro, 11(1), 107-123.

Bolsoni-Silva, A. T., Del Prette, A., \& Del Prette, Z. A. P. (2000). Relacionamento pais-filhos: Um programa de desenvolvimento interpessoal em grupo. Psicologia Escolar e Educacional, 3(3), 203-215.

Bolsoni-Silva, A. T., Salina-Brandão, A., Rosin-Pinola, A. R., \& Versuti-Stoque, F. M. (2008). Avaliação de um programa de intervenção de habilidades sociais educativas parentais: Um estudo-piloto. Psicologia: Ciência e Profissão, 28(1), 18-33.

Bolsoni-Silva, A. T., Silveira, F. F., \& Marturano, E. M. (2008). Promovendo habilidades sociais educativas parentais na prevenção de problemas de comportamento. Revista Brasileira de Terapia Comportamental e Cognitiva, 5(2), 125-142.

Bongers, H. L., Koot, H. M., Ende, J. V. D., \& Verhulst, F. C. (2004). Developmental trajectories of externalizing behaviors in childhood and adolescence. Child Development, 75(5), 1523-1537.

Brandth, B., \& Kvande, E. (2002). Reflexive fathers: Negotiating parental leave and working life. Gender, Work and Organization, 9(2), 186-203.

Caballo, V. E., \& Simón, M. A. (2005). Manual de Psicologia Clínica e do adolescente: Transtornos específicos. São Paulo, SP: Santos.

Carpenter, B. (1997). Early intervention and identification: Finding the family. Children \& Society, 11, 173-182.

Cia, F., \& Barham, E. J. (2008). Trabalho noturno e o novo papel: Uma interface difícil. Estudos de Psicologia (Campinas), 25(2), 213-233.

Cia, F., D’Affonseca, S. M., \& Barham, E. J. (2004). A relação entre envolvimento paterno e desempenho acadêmico dos filhos. Paidéia: Cadernos de Psicologia e Educação, 14(29), 277-286.

Cia, F., Pamplin, R. C. O., \& Del Prette, Z. A. P. (2006). Comunicação e participação pais-filhos: Correlação com habilidades sociais e problemas de comportamento dos filhos. Paidéia: Cadernos de Psicologia e Educação, 16(35), 395-406.

Cia, F., Pereira, C. S., Del Prette, Z. A. P., \& Del Prette, A. (2006). Habilidades sociais parentais e o relacionamento entre pais e filhos. Psicologia em Estudo, 11(1), 73-81.

Coley, R. L. (2001). (In) visible men: Emerging research on low-income, unmarried, and minority fathers. American Psychologist, 56(9), 743-753.

Coley, R. L., Morris, J. E., \& Hernandez, D. (2004). Out-ofschool care and problem behavior trajectories among lowincome adolescents: Individual, family, and neighborhood characteristics as added risks. Child Development, 73(3), 948-965.

Conte, F. C. S. (2001). Promovendo a relação entre pais e filhos. In M. Delitti (Ed.), Sobre comportamento e cognição (pp. 159-166). Santo André, SP: Esetec.

Cooper, B., \& Cooper, N. (2005). What a difference a parent makes! New York: A Focus on Father Workbook to Accompany. 
Cozby, P. C. (2002). Métodos de pesquisa em ciências do comportamento. São Paulo, SP: Atlas.

Crean, H. F. (2008). Conflict in the Latino parent-youth dyad: The role of emotional support from the appositive parent. Journal of Family Psychology, 22(3), 484-493.

Critério Brasil (2006). Associação Brasileira de Empresas de Pesquisa. Retrieved February 05, 2007, from http:// www.abep.org

D’Avila-Bacarji, K. M. G., Marturano, E. M., \& Elias, L. C. S. (2005). Suporte parental: Um estudo sobre crianças com queixas escolares. Psicologia em Estudo, 10(1), 110-115.

Davidov, M., \& Grusec, J. E. (2006). Untangling the links of parental responsiveness to distress and warmth to child outcomes. Child Development, 77(1), 44-58.

Del Prette, Z. A. P., \& Del Prette, A. (2005). Psicologia das habilidades sociais na infância: Teoria e prática. Petrópolis, RJ: Vozes.

Dessen, M. A., \& Szelbracikowski, A. C. (2004). Crianças com problemas de comportamento exteriorizado e a dinâmica familiar. Interação em Psicologia, 8(2), 171-180.

Duch, H. (2005). Redefining parent involvement in Head Start: A two-generation approach. Early Child Development and Care, 175(1), 23-35.

Dunn, J., Cheng, H., O'Connor, T. G., \& Bridges, L. (2004). Children's perspectives on their relationships with their nonresident fathers: Influences, outcomes and implications. Journal of Child Psychology and Psychiatry, 45(3), 553566.

Eisenberg, N., Zhou, Q., Spinrad, T. L., Valiente, C., Fabes, R. A., \& Liew, J. (2005). Relations among positive parenting, children's effortful control, and externalizing problems: A three-wave longitudinal study. Child Development, 76(5), 1055-1071.

Fabiano, G. A. (2007). Father participation in behavioral parent training for ADHP: Review and recommendations for increasing inclusion and engagement. Journal of Family Psychology, 21(4), 683-693.

Fagan, J., \& Iglesias, A. (1999). Father involvement program effects on fathers, father figures, and their Head Start children: A quasi-experimental study. Early Childhood Research Quarterly, 14(2), 243-269.

Fletcher, A. C., Steinberg, L., \& Williams-Wheeler, M. (2006). Parental influences on adolescent problem behavior revisiting Stattin and Kerr. Child Development, 75(3), 781796.

Flouri, E. (2005). Father's involvement and psychological adjustment in Indian and White British secondary school age children. Child and Adolescent Mental Health, 10(1), 32-40.

Flouri, E., \& Buchanan, A. (2003). The role of father involvement in children's later mental health. Journal of Adolescence, 26, 63-78.

Gomide, P. I. C. (2004). Pais presentes pais ausentes: Regras e limites. Petrópolis, RJ: Vozes.

Gravena, A. C. (2006). Retorno ao trabalho após o nascimento de um filho: Percepções de professoras sobre sua experiência. Dissertação de Mestrado não-publicada, Programa de Pós-Graduação em Educação Especial, Universidade Federal de São Carlos, SP.

Gresham, F. M., \& Elliott, S. N. (1990). Social skills rating system. Circle Pines, MN: American Guidance Service.

Hays, W. L. (1981). Statistics ( $3^{\text {rd }}$ ed.). New York: Holt Reinhart e Winston.
Hill, N. E., Castellino, D. R., Lansford, J. E., Nowlin, P., Dodge, K. A., Bates, J. E., et al. (2004). Parent academic involvement as related to school behavior, achievement and aspirations: Demographic variations across adolescence. Child Development, 75(5), 1491-1509.

Hong, S., \& Ho, H. (2005). Direct and indirect longitudinal effects of parental involvement on student achievement: Second-Orde latent growth modeling across ethnic groups. Journal of Educational Psychology, 97(1), 32-42.

Lamb, M. E. (1997). Fathers and child development: An introductory overview and guide. In M. E. Lamb (Ed.), The role of the father in child developmental (pp. 1-18). New York: John Wiley \& Sons.

López, F. (2004). Problemas afetivos e de conduta na sala de aula. In C. Coll, A. Marchesi, \& J. Palácios (Eds.), Desenvolvimento psicológico e educação: Transtornos do desenvolvimento e necessidades educativas especiais (pp. 113128). Porto Alegre, RS: Artmed.

Machado, M. H. S. M. S. (2007). Família e insucesso escolar. Tese de Doutorado não-publicada, Faculdade de Psicologia e de Ciências da Educação, Universidade do Porto, Portugal.

Martínez, A. M. (2007). Inclusão escolar: Desafios para o psicólogo. In A. M. Martinez (Ed.), Psicologia escolar e compromisso social (pp. 95-114). Campinas, SP: Átomo \& Alínea.

Marturano, E. M. (2004). Fatores de risco e proteção no desenvolvimento sócio-emocional de crianças com dificuldades de aprendizagem. In E. G. Mendes, M. A. Almeida, \& L. C. A. Williams (Eds.), Avanços recentes em Educação Especial (pp. 159-165). São Carlos, SP: Editora da Universidade Federal de São Carlos.

Matta, D. S., \& Knudson-Martin, C. (2006). Father responsivity: Couple processes and the coconstruction of fatherhood. Family Process, 45(1), 19-37.

Nascimento, I. M. G. (2007). Investimento no trabalho e na parentalidade e relação interpapéis: Uma análise da transmissão intergeracional. Tese de Doutorado não-publicada, Faculdade de Psicologia e de Ciências da Educação, Universidade do Porto, Portugal.

Olivares, J., Méndez, F. X., \& Ros, M. C. (2005). O treinamento de pais em contextos clínicos e da saúde. In V. E. Caballo \& M. A. Simón (Ed.), Manual de Psicologia Clínica infantil e do adolescente: Transtornos específicos (pp. 365-385). São Paulo, SP: Santos.

Omeño, G. R. (2004). Intervenção com crianças pré-escolares agressivas: Suporte à escola e à família em ambiente natural. Dissertação de Mestrado não-publicada, Programa de Pós-Graduação em Educação Especial, Universidade Federal de São Carlos, SP.

Pacheco, J., Alvarenga, P., Reppold, C., Piccinini, C. A., \& Hutz, C. S. (2005). Estabilidade do comportamento antisocial na transição da infância para a adolescência: Uma perspectiva desenvolvimentista. Psicologia: Reflexão e Crítica, 18(1), 55-61.

Parke, R. D. (2004). Development in the family. Annual Reviews Psychology, 55, 365-399.

Pinheiro, M. I. S., Haase, V. G., Del Prette, A., Amarante, C. L. D., \& Del Prette, Z. A. P. (2006). Treinamento de habilidades sociais educativas para pais de crianças com problemas de comportamento. Psicologia: Reflexão e Crítica, 19(3), 1-15. 
Raley, S. B., Mattlingly, M. J., \& Bianchei, S. M. (2006). How dual are dual-income couples? Documenting change from 1970 to 2001. Journal of Marriage and Family, 68, 11-28.

Rocha, M. M., \& Brandão, M. Z. S. (2001). A importância do autoconhecimento dos pais na análise e modificação de suas interações com os filhos. In M. Delitti (Ed.), Sobre comportamento e cognição (pp. 133-141). Santo André, SP: Esetec.

Stein, L. M. (1994). Teste de desempenho escolar. São Paulo, SP: Casa do Psicólogo.

Taylor, J., \& Daniel, B. (2000). The rhetoric versus the reality in child care and protection ideology and practice, in working with fathers. Journal of Advanced Nursing, 31(1), 12-19.

Tiedje, L. B. (2004). Process of change in work/home incompatibilities employed mothers. Journal of Social Issues, 60(4), 787-800.

Williams, L. C. A., \& Aiello, A. L. R. (2004). O empoderamento de famílias: O que é e como medí-lo. In E. G. Mendes, M. A. Almeida, \& L. C. A. Williams (Eds.), Avanços recentes em Educação Especial (pp. 197-202). São Carlos, SP: Editora da Universidade Federal de São Carlos. 